\title{
Monographie hydrologique du bassin de la Maronne
}

\section{Hydrological Monography of the Maronne basin}

\author{
Travail récompensé par l'attribution du Prix de Monographie Hydrologique \\ de la Société Hydrotechnique de France, pour l'année 1949. \\ PAR GASTON REMENTERAS

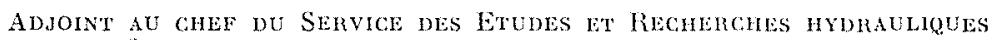 \\ D'Erectricité de France \\ ET JWAN BOYER \\ INGÉNIEUR A LA SOClÉtE HYDROTEChNiQUe DE France
}

English synopsis, p. 767

\section{TROISIEME PARTIE (1)}

\section{CHA P I R E V \\ CAPACITÉ DE RÉTENTION. - COURBE DE TARISSEMENT DU BASSIN}

\section{I. - Régime propre ou non influencé de la Maronne à la station du pont de l'Estouroc.}

On dit qu'une source est en régime propre ou non influencé lorsqu'elle ne recoit aucun apport d'eau extérieur et que son débit $Q$ est uniquement fonction du volume d'eau $V$ accumulé dans les nappes qui l'alimentent : $Q=f(V)$. L'expérience montre que dans ces conditions, si, a un moment donné, il existe un débit $Q_{0}$, la loi de décroissance de ce débit en fonction du temps sera toujours la même. Donc, la détermination des débits d'une source en fonction du temps

(1) La première et la deuxieme parties ont paru respectivement dans les $n^{\prime \prime s} 2$ et 4 de la Houille Blanche (1950). dans la période de non influence ou de régime propre peut s'obtenir par la superposition des courbes de débit de plusieurs années.

Marllet a donné la formule suivante comme expression du débit $Q$ à l'instant $t$ en fonction du débit $Q_{0}$ au temps 0 .

$$
\mathrm{Q}=\mathrm{Q}_{0} e^{-\alpha t}
$$

z est un nombre appelé "coefficient de tarissement $\gg$.

De la formule ci-dessus on tire :

$$
\log \mathrm{Q}=\log \mathrm{Q}_{0}-\alpha \log e \times t
$$

relation qui montre que la courbe représentative de $\log Q$ en fonction de $t$ est une droite. 
Bien que cela constitue une extrapolation quelque peu audacieuse, on peut admettre les mêmes propositions pour l'élude de la décroissance des débits de la Maronne en l'absence de toute pluie ou de toute neige sur son bassin, et en tirer quelques déductions pratiques pour l'exploitation des usines hydro-électriques. La formule de MaILlet ou son équivalent expérimental : « la courbe de tarissement $\gg$ répondent en effet à la question suivante : « Etant donné le débit $Q_{0}$ de la Maronne en un point aujourd'hui, quelle sera sa valeur demain ou dans $n$ jours, s'il ne pleut pas et s'il n'y a ni fonte de neige ni précipitation occulte? $\gg$

Connaitre ainsi la valeur des débils moyens journaliers dans la période de $n$ jours à venir, c'est pouvoir fixer la courbe des puissances $m i$ nima disponibles aux usines pour cette période; s'il pleut suffisamment, le débit de la rivière montera (1) et permettra de fournir davantage d'énergie. Sans doute, le chef d'exploitation trouvera-t-il dans le flair que donment une longue

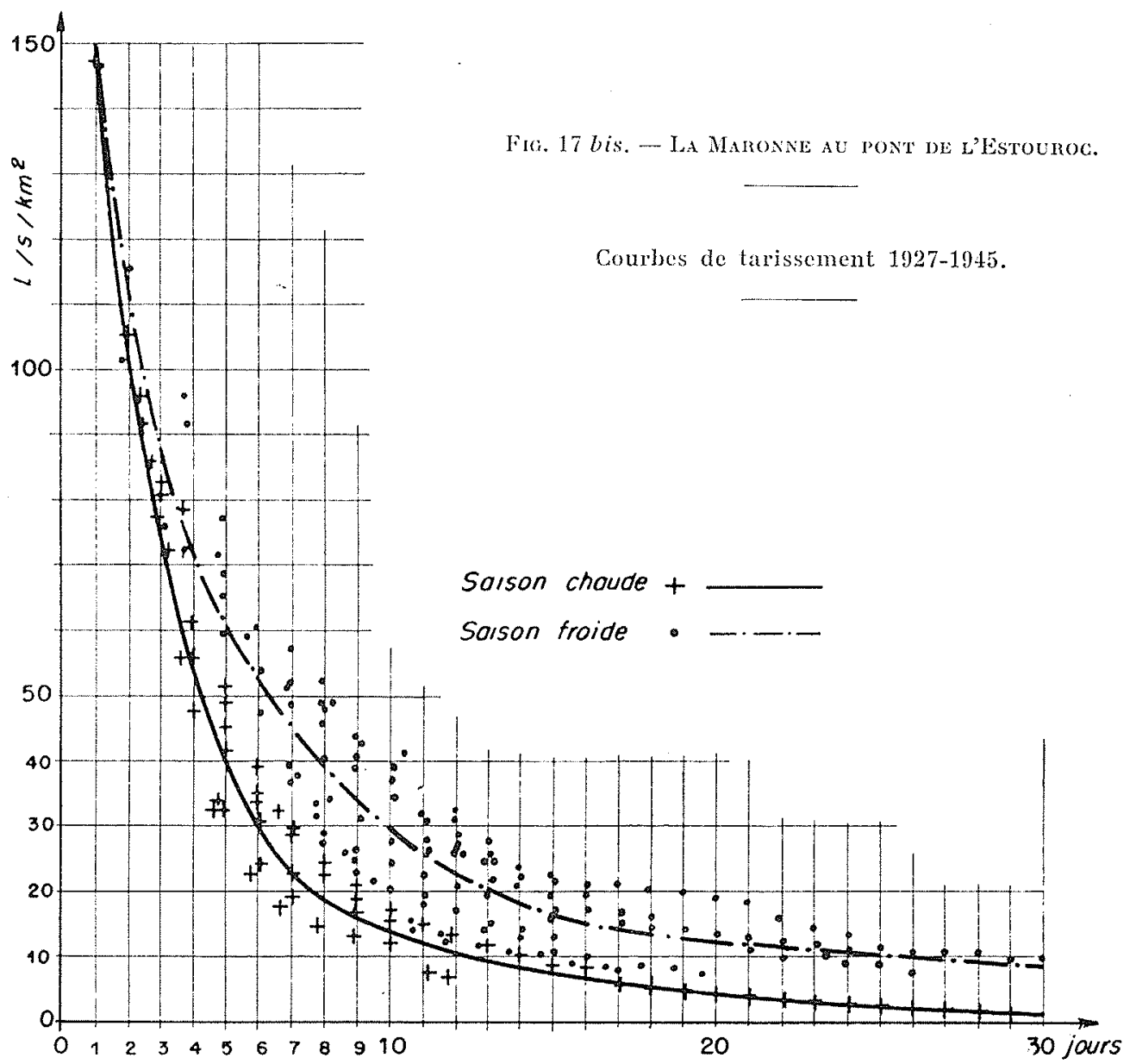

expèrienec et une observation patiente, une méthode de prévision plus précise appuyée sur la situation météorologique générale et sur les plus petits phénomènes météorologiques locaux; nous pensons néanmoins que les courbes de tarissement déterminées comme il est dit ci-après, pourront lui fournir le squelette quasi mathématique de ses prévisions.

Nous avons établi la courbe de tarissement pour la Maronne au pont de l'Estouroc (fig. 17 bis).

Les points sont assez dispersés, ce qui s'explique bien par le caractère approximatif de nos bases théoriques et par la difficulté de savoir si l'on se trouve réellement en régime non influencé. D'autre part, les prévisions ne peuvent être faites qu'à partir d'un certain débit dit « de

(1) On peut d'ailleurs tenter de calculer le débit correspondant à une pluie donnéc si on connait l'intensité des précipitations en fonction du temps. Nous ne croyons pas que ces calculs asse complexes soient d'une grande utilité pratique. Sur la Maronne, en effet, le maximum de la crue suivra de près la fin de la pluie et notre chef d'exploitation pourra connaitre le débit des jours suivants en consultant a ce moment ses courbes de tarissement. 
saturation » qui est le plus fort débit observé en l'absence de toute pluie et après un temps suffisant pour que l'écoulement superficiel ait cessé.

En outre, il est bien évident que les débits décroissent plus rapidement par temps chaud et sec que par temps froid et humide; toutefois, un froid très vif fait baisser les débits en gelant les exutoires des petites sources. Pour tenir compte de ces particularités, nous avons établi deux abaques : l'un pour la saison chaude, maioctobre; l'autre pour Ia saison froide, novembre-avril.

Ces courbes ne constituent d'ailleurs qu'une première approximation, car leur établissement nécessiterait des relevés hydrologiques plus nombreux. Dans le bassin de la Maronne, ou il pleut 135 jours par an en moyenne, il est peu fréquent de trouver une série de 10 à 15 jours sans aucune précipitation, surtout en hiver.

Quoiqu'il en soit, les courbes de tarissement illustrent le caractère très torrentiel de la Maronne : un débit de $72 \mathrm{l} / \mathrm{s} / \mathrm{km}^{2}$ tombe 8 jours après' à $12 \mathrm{l} / \mathrm{s} / \mathrm{km}^{2}$ pendant la saison chaude et à $22 \mathrm{l} / \mathrm{s}$ pendant la saison froide; un débit de $30 \mathrm{l} / \mathrm{s} / \mathrm{km}^{2}$ tombe en 3 jours à $16 \mathrm{l} / \mathrm{s}$ en saison chaude et à $20 \mathrm{l} / \mathrm{s}$ en saison froide.

\section{II. - Capacité de rétention.}

L'intégration de la courbe de tarissement en saison froide :

$$
\int_{t_{0}}^{\infty} \mathrm{Q} d t
$$

donne avec une approximation par défaut (1) le cube d'eau retenu dans le terrain pour le débit $Q_{0}$ correspondant à l'abscisse $t_{0}$. Ce chiffre caractérise la capacité de rétention du bassin et sert à calculer le coefficient d'écoulement vrai (voir chapitre VI).

Pour fixer les idées, disons que le cube d'eau retenu dans le bassin versant de la Maronne au pont de l'Estouroc, lorsque le débit est de

(1) En effet, le cube d'eau retenu dans le terain doit fournir non seulement le débit réellement écoulé par la rivière, mais aussi celui correspondant aux pertes par évaporation.
$30 \mathrm{l} / \mathrm{s}^{2} / \mathrm{km}^{2}$, peut ètre estimé, en saison froïde, à $25.000 \mathrm{~m}^{3}$ par kilomètre carré environ, soit une lame d'eau de $25 \mathrm{~mm}$ d'épaisseur uniformément répartie sur tout le bassin. En période chaude, la courbe en trait plein montre que la réserve « utile » sera de l'ordre de la moitié de la précédente. Pour un débit de $72 \mathrm{l} / \mathrm{s} / \mathrm{km}^{2}$, la capacité de rétention peut être estimée à environ $50.000 \mathrm{~m}^{3}$ par $\mathrm{km}^{2}$, soit une lame d'eau de $50 \mathrm{~mm}$ d'épaisseur uniformément répartie sur tout le bassin, en saison froide.

Il est curieux de constater que, dans cet état de saturation, le volume de l'eau retenue par tout le bassin à l'amont du pont de l'Estouroc ne dépasse pas $26.650 .000 \mathrm{~m}^{3}$, soit un tiers environ de la capacité du réservoir d'Enchanet.

Suivant certains auteur's, la capacité de rétention à un instant déterminé constituerait une mesure des propriétés mécaniques des' sols alluvionnaires du bassin et, par suite, de leur résistance à l'érosion génératrice d'une grande partie du débit solide des cours d'eau correspondants.

\section{III. - Capacité de rétention nivale.}

La rétention nivale joue un certain rôle dans le haut bassin de la Maronne durant les mois d'hiver et de printemps. Les grandes crues de printemps sont le plus souvent dues à des pluies chaudes qui amènent la fonte rapide des neiges.

Ơn pourra se faire une idée du volume d'eau emmagasiné sous forme de neige dans le haut bassin à un instant donné, en admettant que toute précipitation de saison froide au-dessus de l'isotherme de $0^{\circ}$ est tombée sous forme de neige et que le tracé des isothermes et des isohiètes suit celui des courbes de niveau, toutes hypothèses qui ne sont qu'approximatives.

Ce calcul, qui n'a quelque chance d'approcher la réalité que s'il n'y a pas eu de fonte de neige dans la période considérée, ne présentera d'ailleurs d'intérêt que si l'enneignement est important et persistant, circonstances assez rares sur la Maronne; toutefois, étayé par quelques mesures' directes (à la sonde) de l'épaisseur du manteau de neige, il peut fournir à la fin de l'hiver une évaluation du volume d'eau des réserves nivales, élément précieux pour la manœuvre correcte du réservoir saisonnier d'Enchanet. 


\section{CHA PT T R V I}

\section{COEFFICIENT DE RUISSELLEMENT. - DEFICIT D'ÉCOULEMENT}

\section{I. - Définitions.}

Soit $\mathrm{P}$ le module pluviométrique (hauteur de pluie tombée annuellement) et $\mathrm{D}$ le module d'écoulement (hauteur de la lame d'eau qui, uniformément répartie sur le bassin versant, représenterait le volume total débité annuellement par la rivière au point considéré).

La différence $\delta=\mathrm{P}-\mathrm{D}$ qui représente l'eau perdue s'appelle le déficit d'écoulement.

Le rapport $\rho=\frac{\mathrm{D}}{\mathrm{P}}$ est le coefficient de ruissellement apparent.

Pour analyser de plus près les phénomènes, décomposons les termes $D$ et $P$. Le bilan hydraulique du bassin peut être présenté sous la forme suivante :

\begin{tabular}{|c|c|}
\hline PASSIF & ACTIF \\
\hline 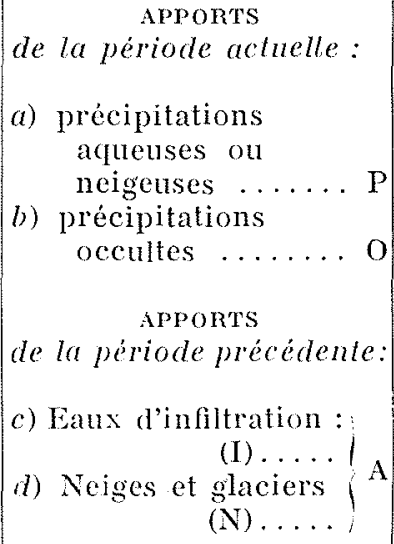 & 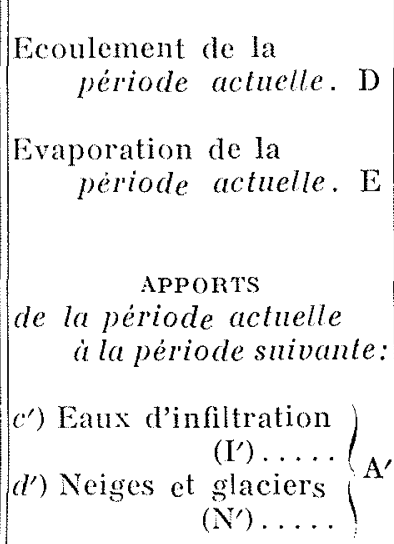 \\
\hline $\mathrm{P}+\mathrm{O}+\mathrm{A}$ & $=\mathrm{D}+\mathrm{E}+\mathrm{A}^{\prime}$ \\
\hline
\end{tabular}

On définit en général le coefficient de ruissellement vrai par le rapport :

$$
r=\frac{\mathrm{D}+\mathrm{A}^{\prime}}{\mathrm{P}+\mathrm{O}+\mathrm{A}}
$$

Si nous admettons que les précipitations oc- cultes sont négligeables en posant $\mathrm{P}+\mathrm{O}=\mathrm{P}$, on montre facilement que l'on a :

$$
r=\mathrm{I}-\frac{\mathrm{E}}{\mathrm{P}+\mathrm{A}} \text { et } \vartheta=\mathrm{I}-\frac{\mathrm{E}}{\mathrm{P}}+\frac{\mathrm{A}-\mathrm{A}^{\prime}}{\mathrm{P}}
$$

et très approximativement :

$$
p=r+\frac{\mathrm{A}-\mathrm{A}^{\prime}}{\mathrm{P}}
$$

Si $\mathrm{A}=\mathrm{A}^{\prime}$, ce que l'on peut admettre en l'absence de neige si les débits de la rivic̀re au début et à la fin de la période considérée sont égaux ou si $\frac{\mathrm{A}-\mathrm{A}^{\prime}}{\mathrm{P}}$ est négligeable, ce qui est réalisé lorsque la période d'observation est assez longue (une année par exemple), on a sensiblement $:=r$.

\section{II. - Calcul du déficit d'écoulement moyen et $\mathrm{du}$ coefficient de ruissellement moyen pour la période 1921-1945.}

\section{Station dU PONT de L'Estouroc}

Pour calculer ces éléments, il est nécessaire de connaître à la fois, pour la période considérée, le volume d'eau roulé en moyenne par la Maronne et celui tombé sur son bassin versant. Pour calculer ce dernier, nous ne disposons que des moyennes pluviométriques relevées à Marmanhac de 1921 à 1945 ('année 1923 manque). Nous avons admis que le rapport entre la pluviosité moyenne sur le bassin de la Maronne au pont de l'Estouroc et la pluviosité à Marmanhac était celui résultant des moyennes de M. Avcio'T (voir chapitre III), soit :

$$
\frac{1.420}{1.197}=1,186
$$

Le tableau XVII donne le calcul des coefficients de ruissellement o et des déficits d'écoulement annuels et moyens pour la période 19211945 .

On voit que, pour la moyenne de cette période le coefficient de ruissellement ressort à $0,593 \mathrm{et}$ 
le déficit hydrologique à $680 \mathrm{~mm}$; pour la période 1921-1930, ces paramètres étaient respectivement : 0,644 et $555 \mathrm{~mm}$.

Ces chiffres semblent un peu anormaux eu egard à ce que l'on sait d'autre part de l'hydrologie de la région. M. Covtagne dome (1) les résultats suivants pour quelques rivières du Massif Central :

\begin{tabular}{|c|c|c|c|c|}
\hline Rivières et stations & $\begin{array}{c}\text { Période } \\
\text { d'observation }\end{array}$ & $\begin{array}{c}\text { Hanteur } \\
\text { des précipitations } \\
\text { P en mm }\end{array}$ & $\begin{array}{c}\text { Coefficient } \\
\text { de ruissellement } \\
\text { moyen }\end{array}$ & $\begin{array}{l}\text { Déficit } \\
\text { hydrologique } \\
\text { en mm }\end{array}$ \\
\hline 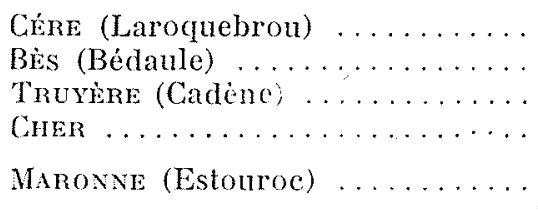 & $\begin{array}{r}1910-1914 \\
1916-1924 \\
1910-1918 \\
1910-1918 \\
1921-1930 \\
1921-1945\end{array}$ & $\begin{array}{r}1.370 \\
1.152 \\
1.127 \\
916 \\
1.593 \\
1.669\end{array}$ & $\begin{array}{l}0,72 \\
0,628 \\
0,56 \\
0,436 \\
0,644 \\
0,593\end{array}$ & $\begin{array}{l}384 \\
427 \\
496 \\
502 \\
555 \\
680\end{array}$ \\
\hline
\end{tabular}

On admet souvent qu'eñ dehors des pluviosités extrêmes le coefficient de ruissellement o varie proportionnellement à la pluviosité. M. Coutane a proposé pour le Massif Central la formule :

$$
p=0,5 \text { à } 0,52 \mathrm{P} \text { ( } \mathrm{P} \text { en mètres })
$$

Il en résulte que le module d'écoulement proportionnel au cube roule annuellement par la rivière serait de la forme :

$$
\mathrm{D}=0,5 \mathrm{P}^{2}
$$

c'est-à-dire que les modules annuels successil's d'une rivière sont proportionnels au carré des pluviosités correspondantes.

Ces relations se vérifient assez mal pour la Maronne; les valeurs moyemnes de p et de D répondraient assez bien à la formule :

$$
\begin{aligned}
? & =0,355 \mathrm{P} \\
\text { et } \mathrm{D} & =0,355 \mathrm{P}^{2}
\end{aligned}
$$

mais ces relations ne s'appliquent pas aux années prises successivement. Peut-être la période considérée comprend-elle trop d'années exceptionnelles (1921-1922-1929-1935-1942); peut-être encore les pluviosités moyennes admises faute d'informations précises, notamment sur la haute Maronne, sont-elles trop élevées ou les débits relevés aux Estourocs estimés par défaut?

Ces divergences ont du moins le mérite de montrer avec quelle prudence il faut interpréter les calculs hydrologiques reposant sur des observations trop précaires.

\section{III. - Variation saisonnière des coefficients de ruissellement. - Coefficients moyens de ruissellement mensuel.}

Nous avons calculé la valeur des coefficients de ruissellement apparents moyens mensuels pour la période 1927-1945. A titre d'exemple, le tableau XVII donne le détail du calcul pour quelques années caractéristiques.

Pour en tirer des conclusions précises au point de vue de l'hydrologie pure, il faudrait calculer les coefficients de ruissellement vrais en tenant compte des rapports d'un mois à l'autre dus aux réserves d'eau emmagasinées dans les nappes souterraines ou dans la couverture nivale. Tel quel, le tableau ci-dessus peut pourtant rendre quelques services, par comparaison avec les relevés contemporains de pluie et de température en montrant la variation du coefficient de ruisseliement en fonction de ces parametres et suivant les saisons.

Ciette notion peut servir en pratique à estimer le cube d'eau qui sera apporté à la riviere par une pluie de hauteur déterminée et à préciser ainsi les données fournies par la « courbe de tarissement $\otimes$.

On voit que, durant les mois d'été, le coefficint de ruissellement peut descendre au-dessous de 0,10 , tandis qu'il peut atteindre 0,75 en

(1) Voir Revale Génerale de l'Electricile, 18 juin 1921, page 855 . Contribution à l'étude du ruissellement et à la détermination du régime hydraulique d'un bassin en fonction de sa pluviosité. 
moyenne pour les pluies de printemps et d'hiver. Les coefficients de ruissellement supérieurs à 1 proviennent de la fonte des neiges tombées durant le mois précédent... ou d'une erreur dans les observations pluviométriques.

D'ailleurs, il ne faut pas se faire d'illusion sur la précision de ces divers coefficients qui sont fonctions d'observations difficiles à contrôler; on doit se borner à rechercher un ordre de grandeur qui donne le sens des phénomènes et per- . met, le cas échéant, d'éliminer une observation manifestement erronée.

Tabefau XVII. - Riviline : Maronne. - Station : Estouroc

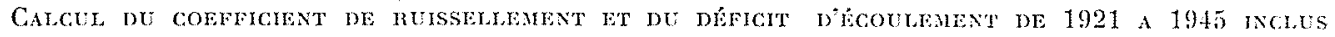

\begin{tabular}{|c|c|c|c|c|c|}
\hline ANNÉES & $\begin{array}{c}\text { HAU'TEUR } \\
\text { de lame d'eau } \\
\text { corlespondant } \\
\text { ati cube roulé } \\
\text { par la Maronne }\end{array}$ & $\begin{array}{c}\text { MAUreur } \\
\text { des précipitations } \\
\text { à Marmanlac }\end{array}$ & $\begin{array}{l}\text { PLuviosité } \\
\text { moyenne } \\
\text { sur l'ensemble } \\
\text { du bassin }\end{array}$ & $\begin{array}{l}\text { COEFFICIENT } \\
\text { de } \\
\text { luissellement }\end{array}$ & $\begin{array}{c}\text { DÉficre } \\
\text { d'econlement }\end{array}$ \\
\hline & D) en $\mathrm{mm}$ & $P^{\prime}$ cn $\mathrm{mm}$ & $\mathrm{P}^{\mathrm{P}}=1.186 \mathrm{P}^{\prime}$ & $\rho=\frac{D}{P}$ & $\hat{o}=\mathrm{P}-\mathrm{D}$ \\
\hline 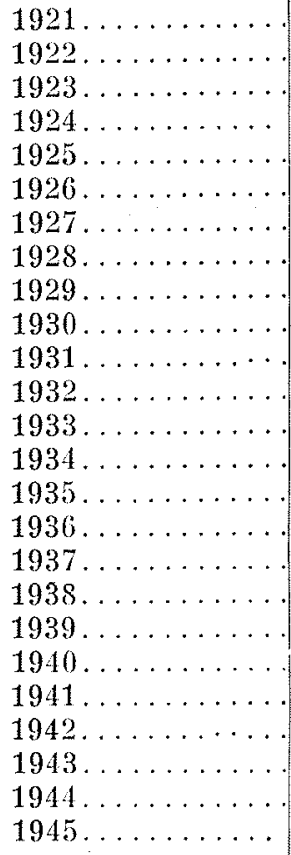 & $\begin{array}{c}470 \\
1.658 \\
? \\
785 \\
783 \\
1.218 \\
1.245 \\
1.129 \\
856 \\
1.228 \\
1.057 \\
1.147 \\
588 \\
1.024 \\
1.323 \\
990 \\
1.099 \\
627 \\
1.128 \\
1.149 \\
1.244 \\
569 \\
604 \\
1.169 \\
658\end{array}$ & $\begin{array}{c}768 \\
1.698 \\
? \\
918 \\
1.053 \\
1.521 \\
1.612 \\
1.542 \\
1.419 \\
1.742 \\
1.422 \\
1.704 \\
1.156 \\
1.544 \\
1.788 \\
1.409 \\
1.706 \\
1.174 \\
1.463 \\
1.574 \\
1.466 \\
1.127 \\
1.352 \\
1.571 \\
1.050\end{array}$ & $\begin{array}{c}910 \\
2.013 \\
? \\
1.088 \\
1.248 \\
1.803 \\
1.912 \\
1.829 \\
1.683 \\
2.067 \\
1.687 \\
2.021 \\
1.372 \\
1.832 \\
2.121 \\
1.672 \\
2.023 \\
1.393 \\
1.736 \\
1.867 \\
1.739 \\
1.337 \\
1.604 \\
1.864 \\
1.246\end{array}$ & $\begin{array}{c}0,516 \\
0,823 \\
? \\
0,721 \\
0,627 \\
0,675 \\
0,051 \\
0,617 \\
0,509 \\
0,594 \\
0,627 \\
0,568 \\
0,429 \\
0,559 \\
0,624 \\
0,592 \\
0,543 \\
0,465 \\
0,650 \\
0,615 \\
0,715 \\
0,426 \\
0,377 \\
0,627 \\
0,528\end{array}$ & $\begin{array}{c}440 \\
355 \\
? \\
303 \\
465 \\
585 \\
667 \\
700 \\
827 \\
839 \\
630 \\
874 \\
784 \\
808 \\
798 \\
682 \\
924 \\
766 \\
608 \\
718 \\
495 \\
768 \\
1.000 \\
695 \\
588\end{array}$ \\
\hline Moyennes....... & 989 & 1.407 & 1.669 & 0,593 & 680 \\
\hline
\end{tabular}




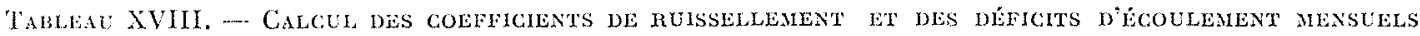

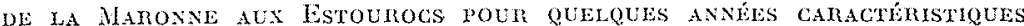

\begin{tabular}{|c|c|c|c|c|c|c|}
\hline ANNÉE & mors & $\begin{array}{l}\text { HAu'reur } \\
\text { de lame d'eau } \\
\text { correspondant } \\
\text { au cube roulé }\end{array}$ & $\begin{array}{c}\text { MAUTEUR } \\
\text { des précipitations } \\
\text { a Marmanhac }\end{array}$ & $\begin{array}{l}\text { PLuvioside } \\
\text { moyenne } \\
\text { sur l'ensemble } \\
\text { du bassin }\end{array}$ & $\begin{array}{l}\text { COEFFICIENT } \\
\text { de } \\
\text { ruissellement }\end{array}$ & $\begin{array}{l}\text { DÉrCir } \\
\text { d'écoulement } \\
\text { (arrondi) }\end{array}$ \\
\hline & & $\mathrm{D}$ en $\mathrm{mm}$ & $\mathrm{P}^{\prime}$ en $\mathrm{mm}$ & $\mathrm{P}=1,186 \mathrm{P}^{\prime}$ & $\rho=\frac{D}{P}$ & $\delta=\mathrm{P}-\mathrm{D}$ \\
\hline 1928. & $\begin{array}{l}\text { Janvier. } \\
\text { Février. } \\
\text { Mars.... } \\
\text { Avril... } \\
\text { Mai..... } \\
\text { Jain.... } \\
\text { Juillet. } \\
\text { Août.... } \\
\text { Septemb. } \\
\text { Octobre. } \\
\text { Novemb. } \\
\text { Décemb. }\end{array}$ & $\begin{array}{r}121,5 \\
189,1 \\
118,9 \\
220,0 \\
125,4 \\
43,5 \\
5,6 \\
4,2 \\
4,7 \\
18,6 \\
185,2 \\
81,5\end{array}$ & $\begin{array}{c}104 \\
169,5 \\
194,7 \\
245,7 \\
114,6 \\
69,3 \\
23 \\
68,7 \\
01,5 \\
134 \\
209,1 \\
105,8\end{array}$ & $\begin{array}{r}123,3 \\
201,0 \\
230,9 \\
291,4 \\
135,9 \\
82,2 \\
27,3 \\
81,5 \\
61,1 \\
158,9 \\
307,3 \\
125,5\end{array}$ & $\begin{array}{l}0,985 \\
0,941 \\
0,515 \\
0,755 \\
0,922 \\
0,529 \\
0,205 \\
0,052 \\
0,077 \\
0,117 \\
0,603 \\
0,649\end{array}$ & $\begin{array}{r}2 \\
12 \\
112 \\
71 \\
11 \\
39 \\
22 \\
77 \\
56 \\
140 \\
122 \\
44\end{array}$ \\
\hline 1929. & $\begin{array}{l}\text { Janvier. } \\
\text { Fevrier. } \\
\text { Mars.... } \\
\text { Avril ... } \\
\text { Mai.... } \\
\text { Juin... } \\
\text { Juillet. } \\
\text { Août.... } \\
\text { Septemb. } \\
\text { Octobre. } \\
\text { Novemb. } \\
\text { Décemb. }\end{array}$ & $\begin{array}{r}73,8 \\
47,1 \\
38,7 \\
39,8 \\
154,6 \\
70,6 \\
18,6 \\
12,3 \\
8,1 \\
80,4 \\
114,1 \\
201,6\end{array}$ & $\begin{array}{c}40 \\
56,5 \\
19 \\
121 \\
248,1 \\
93,5 \\
102 \\
62,5 \\
101 \\
219,5 \\
135,6 \\
217,5\end{array}$ & $\begin{array}{r}47,4 \\
67,0 \\
22,5 \\
143,5 \\
294,2 \\
110,9 \\
121,0 \\
74,1 \\
119,8 \\
260,3 \\
160,8 \\
258,0\end{array}$ & $\begin{array}{l}1,557 \\
0,703 \\
1,720 \\
0,277 \\
0,525 \\
0,637 \\
0,154 \\
0,166 \\
0,068 \\
0,309 \\
0,710 \\
0,781\end{array}$ & $\begin{array}{r}-26 \\
20 \\
-16 \\
104 \\
140 \\
40 \\
102 \\
62 \\
112 \\
180 \\
47 \\
56\end{array}$ \\
\hline 1941. & $\begin{array}{l}\text { Janvier. } \\
\text { Février. } \\
\text { Mars.... } \\
\text { Avril... } \\
\text { Mai. .... } \\
\text { Juin ... } \\
\text { Juillet. } \\
\text { Aon̂t... } \\
\text { Septemb. } \\
\text { Octobre. } \\
\text { Novemb. } \\
\text { Décemb. }\end{array}$ & $\begin{array}{r}235,6 \\
245,9 \\
181,4 \\
131,7 \\
108,5 \\
132,5 \\
17,3 \\
53,6 \\
17,8 \\
14,4 \\
58,4 \\
46,9\end{array}$ & $\begin{array}{r}186,8 \\
191,4 \\
141,2 \\
101,1 \\
149,7 \\
142,0 \\
98,9 \\
159,2 \\
56,5 \\
57,2 \\
137,5 \\
43,8\end{array}$ & $\begin{array}{r}221,5 \\
227,0 \\
167,5 \\
119,9 \\
177,5 \\
168,4 \\
117,3 \\
188,8 \\
67,0 \\
67,8 \\
163,1 \\
51,9\end{array}$ & $\begin{array}{l}1,064 \\
1,083 \\
1,083 \\
1,098 \\
0,611 \\
0,787 \\
0,147 \\
0,284 \\
0,266 \\
0,212 \\
0,358 \\
0,904\end{array}$ & $\begin{array}{r}-14 \\
-19 \\
-14 \\
-12 \\
69 \\
36 \\
100 \\
135 \\
49 \\
53 \\
105 \\
5\end{array}$ \\
\hline 1942 & $\begin{array}{l}\text { Janvier. } \\
\text { Février. } \\
\text { Mars... } \\
\text { Avril... } \\
\text { Mai..... } \\
\text { Juin.... } \\
\text { Juillet. } \\
\text { Août... } \\
\text { Septemb. } \\
\text { Octobre. } \\
\text { Novemb. } \\
\text { Décenb. }\end{array}$ & $\begin{array}{r}70,7 \\
66,5 \\
159,4 \\
57,4 \\
47,5 \\
54,5 \\
15,4 \\
6,4 \\
7,3 \\
14,4 \\
40,7 \\
29,5\end{array}$ & $\begin{array}{r}167,2 \\
75,4 \\
113,7 \\
60,5 \\
151,6 \\
116,3 \\
34,1 \\
75,1 \\
98,9 \\
99,2 \\
53,8 \\
81,0\end{array}$ & $\begin{array}{r}198,3 \\
89,4 \\
134.8 \\
71,8 \\
179,8 \\
137.9 \\
40,4 \\
89,1 \\
117,3 \\
117,7 \\
63.8 \\
96,1\end{array}$ & $\begin{array}{l}0,357 \\
0,744 \\
1,182 \\
0,799 \\
0,264 \\
0,395 \\
0,381 \\
0,072 \\
0,062 \\
0,122 \\
0,638 \\
0,307\end{array}$ & $\begin{array}{r}128 \\
23 \\
-25 \\
14 \\
132 \\
83 \\
25 \\
83 \\
110 \\
103 \\
23 \\
67\end{array}$ \\
\hline
\end{tabular}




\section{ÉTUDE DES CRUES}

Il est à peine besoin de souligner l'importance de l'étude des crues en vue de la réalisation d'un aménagement hydro-électrique et de travaux de protection contre les inondations. Une estimation trop faible de la crue maximum accroît le risque de destruction des ouvrages tandis que l'excès contraire conduit à un supplément de travaux hors de proportion avec l'augmentation de sécurité réalisée.

Le plus souvent, on prend comme base de l'es- timation du plus fort débit à prévoir, le débit de la crue dite « historique » affecté d'un certain coefficient de sécurité. Or, la crue « historique »n'a évidemment pas été jaugée et son évaluation repose sur des supputations fort imprécises. Plusieurs auteurs ont essayé d'établir des formules mathématiques permettant de calculer la crue qui ne sera jamais dépassée (ou mieux, de probabilité très faible) à partir des crues observées durant une série d'annẻes. Ces

Tableau XIX. - Caractéristigues des principales crues de la Maronne de 1918 a 1928 d'après les courbes de débits journaliers au Pont de l'Estouroc et calculées pour un bassin versant de $591 \mathrm{~km}^{2}$ (Maronne + Riou-Tort)

Les crues ci-après indiquées sont celles dont le débit dépasse deux fois le module

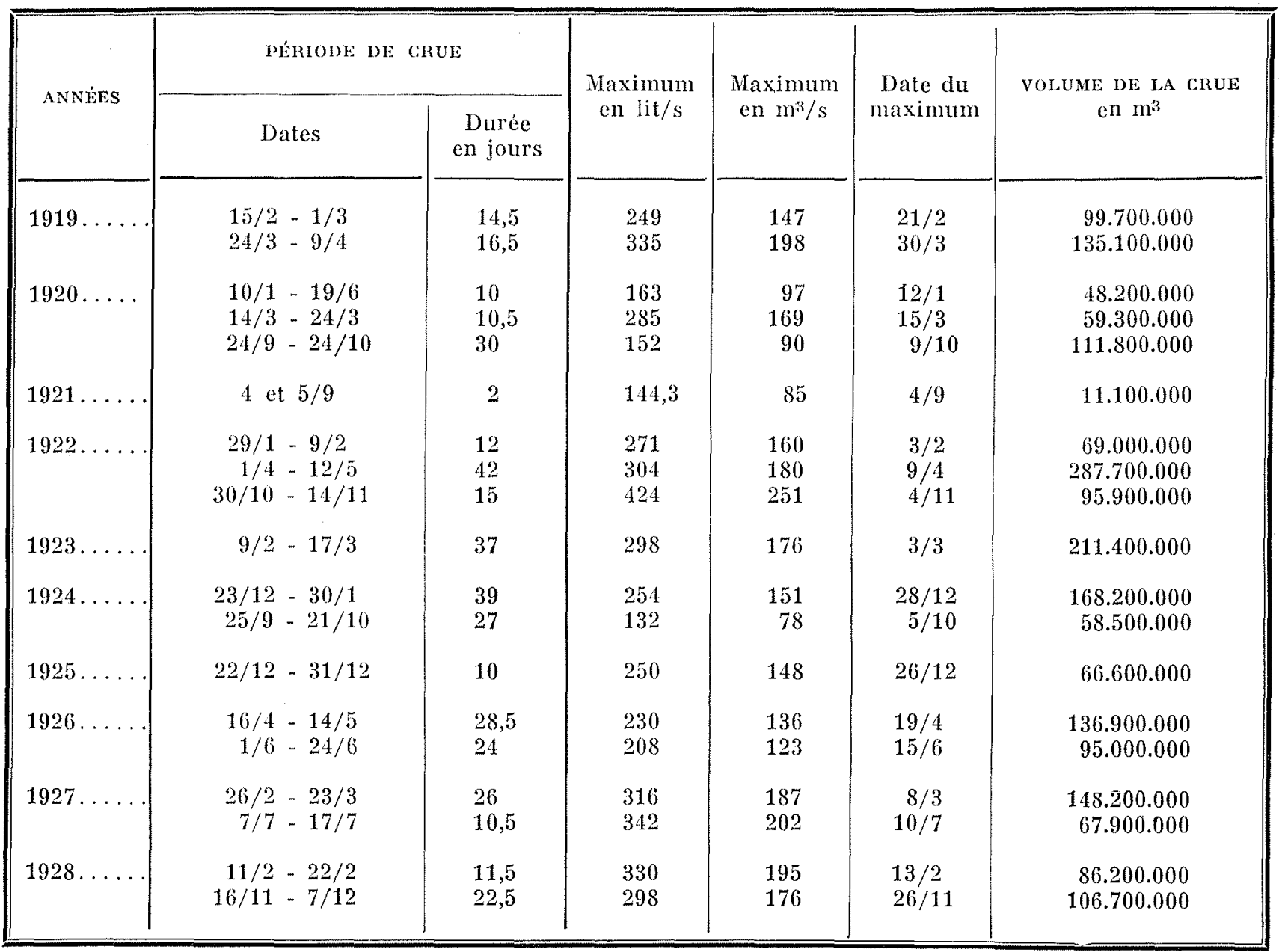


méthodes, malgré leur caractère empirique que nous soulignerons plus loin, permetlent d'obtenir des chiffres de signification assez précise capable d'aider le jugement de l'ingénicur; pour chaque cas particulier, elles donnent, sous certaines conditions, des débits de probabilité comparable; il appartiendra au maitse de l'oxuvre dappliquer à ceux-ci un coefficient de sécurité adéquat en étudiant la réduction du risque, procurée par un aceroissement déterminé des investissements. En pareille matière, le choix dé borde évidemment le cadre de la technique pure!

\section{1. - Crues observées.}

Le tableau XIX donne les caractéristiques des principales crues de la période 1918-1928, pour un bassin versant de $591 \mathrm{~km}^{2}$, d'après les rele- vés de débil à la station du pont de l'Essouroc. Le débit maximum observé durant celte période a été celui du 4 novembre 1922 qui a alteint $424 \mathrm{l} / \mathrm{s} / \mathrm{km}^{2}$. Ce chifre résulte de l'extrapolation linéaire du bareme de la station du pont de l'Estouroc; nous sommes donc portés à croire que le débit réel a été sensiblement plus élevé. On remarquera le cube d'eau considérable roulé par les grandes crues : une période de très hautes eaux d'une quinzaine de jours apporte parfois un volume d'eau supérieur à celui du réservoir saisonnier d'Enchanet.

Durant la période 1928-1944, le débit de $424 \mathrm{l} / \mathrm{s} / \mathrm{km}^{2}$ a été dépassé plusieurs fois, ainsi que l'indique le tableau XX établi par M. BirRIÈre, Ingénieur à la $4^{\circ}$ Circonseription Electrique à Limoges, pour étudier les conditions de fonctionnement des évacuateurs de crue du bar-

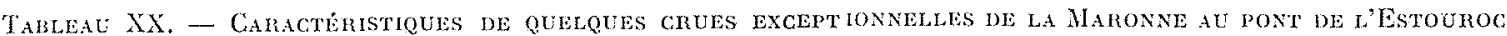
(B.V. $\left.=532,7 \mathrm{~km}^{2}\right)$

\begin{tabular}{|c|c|c|c|c|c|c|c|c|c|c|}
\hline \multirow{3}{*}{$\mathrm{N}^{\circ}$ et Date } & \multicolumn{7}{|c|}{ MONTÉE TOTALE } & \multicolumn{3}{|c|}{ MONTÉE PRÉCÉDANT LE MAX. } \\
\hline & \multicolumn{2}{|c|}{$\begin{array}{c}\text { Hauteur } \\
\text { en } \mathrm{cm}\end{array}$} & \multicolumn{2}{|c|}{$\begin{array}{l}\text { Débit } \\
\text { en } 111^{3} / \mathrm{s}\end{array}$} & \multirow{2}{*}{ Durée } & \multicolumn{2}{|c|}{$\begin{array}{l}\text { Accroissement } \\
\text { moyen de: }\end{array}$} & \multicolumn{2}{|c|}{$\begin{array}{c}\text { Accroissement } \\
\text { moyen de: }\end{array}$} & \multirow{2}{*}{ Durée } \\
\hline & Initiale & Max. & Initial & Max. & & $\begin{array}{c}\text { Hautemr } \\
\text { en cm } \\
p^{*} \text { heure }\end{array}$ & $\begin{array}{c}\text { Débit } \\
\text { en } \mathrm{m}^{3} / \mathrm{s} \\
\mathrm{p}^{\mathrm{r}} \text { heure }\end{array}$ & $\begin{array}{l}\text { Hauteur } \\
\text { en cm } \\
\mathrm{D}^{\mathrm{r}} \text { heure }\end{array}$ & $\begin{array}{c}\text { Débit } \\
\text { en m m/s } \\
\mathrm{P}^{\text {r heure }}\end{array}$ & \\
\hline 1. - 2-12-35. & 150 & 274 & 88 & 235 & $25 \mathrm{~h}$ & 5 & 5,9 & 10 & 13 & $3 \mathrm{~h}$ \\
\hline 2. $-10-12-40$. & 136 & 340 & 78 & 338 & $21 \mathrm{~h}$ & 9,7 & 12,4 & 14,7 & 17 & $6 \mathrm{~h}$ \\
\hline $3 .-14-1-43$ & 143 & 300 & 83 & 272 & $15 \mathrm{~h}$ & 10,5 & 12,6 & 25 & 33 & $2 \mathrm{~h}$ \\
\hline 4. - 21-10-44. & 198 & 320 & 50 & 304 & $24 \mathrm{~h}$ & 9,2 & 10,6 & 18 & 24,5 & 6 h 30 \\
\hline $5,-14-11-44$. & 110 & 288 & 59 & 255 & $20 \mathrm{~h}$ & 8,9 & 9,8 & 19 & 21,5 & $6 \mathrm{~h}$ \\
\hline $6,-8-12-44$ & 111 & 370 & 60 & 392 & $34 \mathrm{~h}$ & 7,6 & 9,4 & 17,4 & 22 & $3 \mathrm{~h}$ \\
\hline 7. - 11-12-44. & 122 & 278 & 68 & 240 & $18 \mathrm{~h}$ & 8,7 & 9,6 & 16,7 & 20,7 & 5 h 30 \\
\hline
\end{tabular}

rage d'Enchanet. Le plus fort débit instantané observé semble être celui correspondant au maximum de la crue du 8 décembre 1944 qui a atteint au pont de l'Estouroc un débit de $392 \mathrm{~m}^{3} / \mathrm{s}$, soit $740 \mathrm{l} / \mathrm{s} / \mathrm{km}^{2}$.

Le tableau XX fournit quelques données numériques sur la vitesse de montée du plan d'eau $\frac{d h}{d t}$ et sur l'accroissement du débit $\frac{d \mathrm{Q}}{d t}$ pendant les grandes crues; ces parametres, qui jouent un ròle important dans l'étude de l'ćcoulement non permanent dans les cours d'eau, ont atteint au pont de l'Estouroc des valeurs maxima de $25 \mathrm{~cm}$ par heure et $33 \mathrm{~m}^{3} / \mathrm{s}$ par heure respectivement.
Pour donner une idée de la forme des ondes de crue, les figures 18 et 19 reproduisent les relevés limnigraphiques relatifs à trois crues caractéristiques :

- La figure 18 représente la crue du 9 au 14 décembre 1940, au pont de l'Estouroc, très violente, brutale et de courte durée, elle est due moins à des précipitations exceptionnelles qu'à une fonte rapide des neiges couvrant le haut bassin;

- La figure 19 représente l'enregistrement au pont de l'Estouroc d'une part, à SainteEulalie d'autre part, de la crue du 9 au 15 janvier 1943 , provoquée par plusieurs jours de pluies abondantes. 
Lat monte des eaux de la Maronne suit de très près le commencement de la pluie. La décrue est notablement plus lente que la crue, ce qui indique une certaine capacité de rétention du bassin. On remarque toujours dans les grandes crues plusieurs maxima successifs; lorsque ceux-ci ne sont pas dus à une recrudescence de la pluie, il faut y voir le passage des maxima

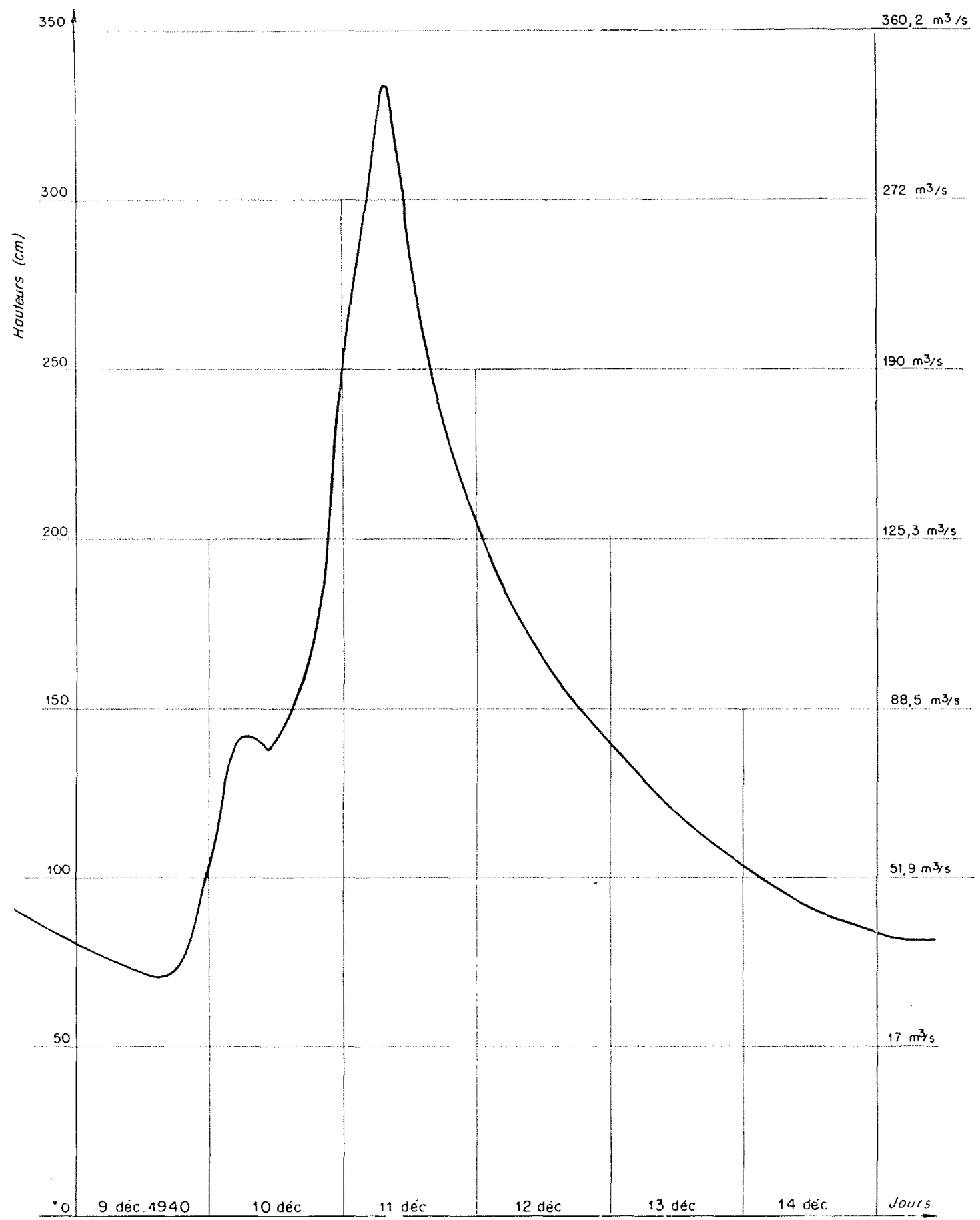

Fig. 18. -- La Maronne au pont de i’Estouroc. - Crue du 9-14 décembre 1940.

successifs des crues des grands affluents. Le premier maximum pourrait correspondre à celui du débit de l'Etze dont le très important bassin en forme d'éventail doit conduire à une propa- gation rapide de l'onde de crue. Toutefois, les premicrs calculs que nous avons effectués pour vérifier cette hypothèse ne nous ont conduits à aucun résultat bien net. L'Encon, bien connu 
pour ses crues rapides et importantes doit, malgré son bassin versant relativement faible, con- tribuer fortement à la montée rapide du flot au pont de l'Estouroc.

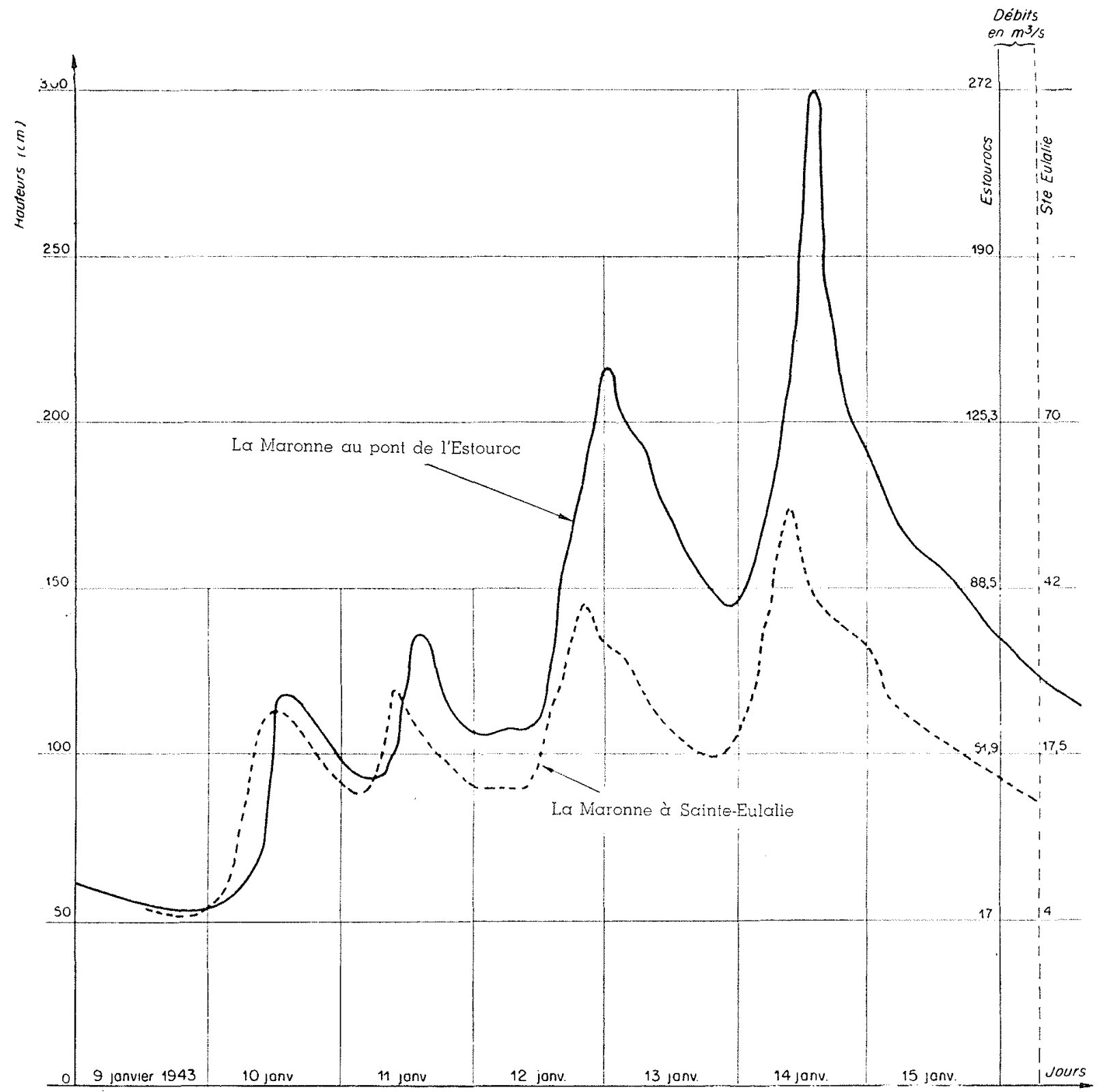

Fig. 19. - La Manonne : Crue du 9-15 janvier 1943 au pont de l'Estouroc et à Sainte-Eulalie.

\section{II. - Application des formules mathéma-} tiques donnant les débits de crue.

a) Variation du débit maximum de crue avec l'importance du bassin versant.

Fuller avait donné la formule (1) :

$$
\mathrm{M}=c \mathrm{~S}^{0,8}
$$

(1) On ajoute quelquefois un deuxième terme a cette formule qui devient ainsi :

$$
\mathrm{M}=\mathrm{cS} 0, \mathrm{~s}+\mathrm{DS} 0,5
$$

$M$ est la valeur moyenne sur une longue série d'années dy débit maximum de la crue annuelle en $1 / \mathrm{s} / \mathrm{km}^{2}$ de bassin versant;

S la superficie du bassin versant;

$c$ une constante qui dépend des caractéristiques du bassin et qui varierait selon les études de Fulker (qui ne s'appliquent qu'à l'Amérique) de 0,6 à 244 !

M. Coutagne propose la formule :

$$
\mathrm{M}=10 \text { à } 50 \sqrt{\mathrm{S}}
$$


la formule $M=20 \vee \bar{S}$ correspondrait généralement à 《la réalité des faits ». Pour la Maronne au pont de l'Estouroc, elle donne :

$$
\mathrm{M}=20 \sqrt{532,8}=461 \mathrm{l} / \mathrm{s} / \mathrm{km}^{2},
$$

valeur qui semble un peu élevée pour la moyenne des crues annuelles (maximum observé :

$\left.740 \mathrm{l} / \mathrm{s} / \mathrm{km}^{2}\right)$.

b) Variation du débit de crue dans le temps. Calcul de la crue décennale, centenaire, millénaire.

Fuller a préconisé la formule :

$$
\mathrm{M}_{\mathrm{L}}=q_{1}\left(1+0,8 \log _{10} \mathrm{~T}\right)
$$

$\mathrm{M}_{r_{1}}$ est le débit de la crue maximum moyenne (et non médiane), correspondant à une période de $\mathbf{T}$ années, et $q_{1}$ la moyenne des débits des crues maxima constatées chaque année.

On peut tenter d'évaluer le débit de la crue survenant une fois en cent ans ou une fois en mille ans en admettant, par une extrapolation toujours risquée, que la distribution statistique des débits sur une longue période est représentée par une courbe algébrique s'ajustant aussi bien que possible à la série toujours limitée des observations disponibles. Dès $1920, M$. Coutagne avait proposé d'assimiler la courbe des débits classés à une parabole de degré $n$ (distribution parabolique), puis plus tard à une exponentielle; dans ce dernier cas, si l'on porte en ordonnée le logarithme du débit et en abscisse le temps', ou mieux la probabilité d'un débit inférieur à celui figurant en ordonnée, on obtient une droite (ce qui est bien la courbe la mieux adaptée aux extrapolations!). M. Gibrat a admis une distribution suivant une courbe de Galton qui correspond à une distribution gaussienne des logarithmes des débits.

La forme mathématique de ces méthodes ne doit pas faire oublier leur caractère purement empirique (interprétation algébrique d'une portion limitée de courbe expérimentale), et la nécessité, avant de les utiliser, de vérifier dans la faible mesure $\mathrm{du}$ possible si elles s'adaptent bien au cas particulier envisagé. Leur adaptation, généralement bonne pour les débits moyens, - les plus fréquents, - devient sans doute de plus en plus précaire et en tout cas de plus en plus difficile à vérifier pour les débits extrêmes - très peu fréquents - tels que ceux afférents aux crues catastrophiques et aux plus bas étiages. Pour en tirer le meilleur parti, l'ingénieur devra s'assurer le double concours d'un hydrologue et d'un statisticien expérimentés.

Quoiqu'il en soit, nous allons, à titre d'exem- ple, faire une application de la formule suivante analogue à celle de Fuller :

$$
\mathbf{M}_{\mathrm{T}}=\alpha(1+\beta \log \mathbf{T})
$$

$\alpha$ et $\beta$ sont deux constantes que l'on calcule graphiquement en tracant la courbe $\mathrm{M}_{\mathrm{T}}=f(\mathrm{~T})$ d'après les valeurs de $M_{T}$ et de $T$ résultant des observations effectuées pendant une durée suffisante.

Pour la Maronne au pont de l'Estouroc les débits de crue observés de 1918 à 1932 se groupent médiocrement autour d'une droite moyenne répondant à l'équation :

$$
\mathrm{M}_{\mathrm{T}}=205\left(1+0,523 \log _{e} \mathrm{~T}\right)
$$

M. Coutagne a obtenu de son côté les formules suivantes :

Cère à Laroquebrou . . $\mathrm{M}_{\mathrm{T}}=200\left(1+0,8 \log _{e} \mathrm{~T}\right)$

Truyère à la Cadène. $\mathrm{M}_{\mathrm{T}}=83\left(1+1,7 \log _{e} \mathrm{~T}\right)$

Tarn au Pinet $\ldots . \quad \mathrm{M}_{\mathrm{T}}=140\left(1+1,1 \log _{e} \mathrm{~T}\right)$

Toutes ces' équations reposent sur un trop petit nombre d'observations pour fournir des résultats bien cohérents. Signalons toutefois que, d'après la formule 1 , la valeur de la crue centenaire (1) au pont de l'Estouroc serait de $700 \mathrm{l} / \mathrm{s} / \mathrm{km}^{2}$. La crue millénaire atteindrait $945 \mathrm{l} / \mathrm{s} / \mathrm{km}^{2}$.

Notre calcul étant basé sur des débits moyens' journaliers de crue, ces chiffres représentent aussi des débits moyens journaliers. Pour obtenir le débit maximum instantané, on peut utiliser la formule suivante due à Fuller :

$$
q_{m}=q\left(1+\frac{2,66}{\mathrm{~A}^{0,8}}\right)
$$

$q_{m}$ est le débit maximum instantané de la crue, $q$ le débit moyen journalier et A la surface du bassin versant en $\mathrm{km}^{2}$.

Pour la Maronne au pont de l'Estouroc, cette formule donne (2):

$$
q_{m}=1,4045 q \text {. }
$$

Les débits maximum maximorum instantanés seraient done de :

$983 \mathrm{l} / \mathrm{s}$ par $\mathrm{km}^{2}$ pour la crue centenaire et $1.327 \mathrm{l} / \mathrm{s}$ par $\mathrm{km}^{2}$ pour la crue millénaire.

(1) C'est-à-dire, en langage ordinaire, la crue survenant une fois dans une période de cent ans.

(2) A titre de comparaison on a observé : pour la crue $d u 1^{\text {er }}$ au 2 décembre 1932

(fonte de neige) .................. $q_{m}=1,32 q$ pour celle du $4-5$ avril 1932 (pluies) ......... $q_{m}=1,14 q$ pour celle du 2-3 juin 1932 (pluies).......... $q_{m}=1,37 q$ 
M. Couthane signale que le débit de la crue centenaire est compris entre 2 et 2,5 fois colui de la crụe de probabilité $1 / 2$ (crue dépassée en moyenne un an sur deux). Pour la Maromne au pont de l'Estouroc, ce dernier débit est égal à 275 litres, de sorte que la crue centenaire est ici égale à :

$\frac{700}{275}=2,54$ fois le débit de la crue de probabilité $1 / 2$.

\section{CHAPITIE VIII}

\section{EVOLUTION DU LIT ET DEBIT SOLIDE}

Le profil en long de la Maronne est sans doute assez proche d'un profil d'équilibre d'allure générale parabolique (voir fig. 2). Il peut grosso-modo être divisé en deux sections; dans la section amont, la rivière coule sur la roche en place qu'elle érode peu à peu jusqu'à l'aval du hameau de Lesturgie; au-delà (section aval) son lit semble en cours de remblaiement; l'épaisseur des alluvions croît progressivement jusqu'au confluent où elle a été estimée à 8 à 12 mètres.

Bien entendu, il ne s'agit là que d'une vue d'ensemble schématique. Si l'on entre dans le détail, on trouvera de petites plaines alluviales dans le haut bassin vers Sainte-Eulalie et Fontange et aussi aux abords de Rodomont. Il n'est pas exclu d'autre part qu'il existe quelques seuils rocheux sur la Maronne inférieure bien à l'aval de la barre du Carlat, mais les nombreuses recomnaissances que nous avons faites et les quelques sondages exécutés dans cette dernière région montrent nettement un remblaiement généralisé et suggèrent parfois l'existence d'un lit épigénétique sous les alluvions.

Aucune mesure de débit solide n'a été effectuée sur la Maronne. Les atterrissements qui ne manqueront pas de se former dans les réservoirs des usines hydroélectriques récemment construites permettront sans doute, dans une dizaine d'années, d'avoir une idée de son importance.

A priori, on peut penser que dans ce pays, géologiquement vieux, le volume des sables et graviers transportés, tant par charriage sur le fond qu'en suspension dans l'eau, est relativement faible. Sauf au voisinage du confluent, on n'observe pas de banes de sables ou de graviers importants et même dans les zones à faible pente on ne voit aucune trace de ces modifications brutales du lit si fréquentes dans les torrents des Alpes.

Dans les régions à forte pente, le lit est constitué soit par la roche en place soit par un pavage de gros blocs qui ne doivent être déplacés que par des crues exceptionnelles. Les secteurs à faible pente offrent des épanouissements for- mant champ d'inondation sur lesquels se déposent les matériaux fins. Durant les crues ordinaires, ces matériaux sont transportés en suspension en assez grande quantite pour rendre l'eau completement opaque; ce sont eux qui formeront la majeure partie des dépôts dans le réservoir saisonnier dEnchanet; le volume des matériaux apportés par charriage sera sans doute insignifiant.

On peut estimer d'une facon assez grossicre le débit solide charrié par la Maronne d'après la cubature des dépôts constatés dans le réservoir de la petite Rhue - sous-affluent de la Dordogne, assez comparable à la haute Maronne - en septembre 1938 (renseignements communiqués par M. Barrit̀re, ingénieur à la $4^{\circ}$ Circonscription électrique à Limoges).

Les principales caractéristiques du réservoir de la petite Rhue alimentant l'usine de Coindre sont les suivantes :

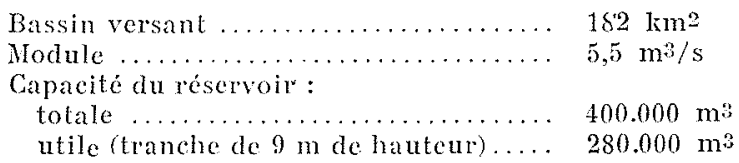

A l'amont de la retenue, les dépôts constitués sans doute en majeure partie par des matériaux charriés sur le fond comportent $8 \%$ \% de sables et de graviers et $15 \%$ de vase et d'éléments végétaux. A proximité du barrage, on trouve $75 \%$ de vase et d'éléments végétaux et $25 \%$ de sables et de graviers; ces matériaux ont été probablement transportés en suspension ou par l' «under-flow $\gg$.

D'après les métrés, on a estimé crue pendant les onze premières années d'exploitation la retenue a recu $60.000 \mathrm{~m}^{3}$ de matériaux dont :

$$
\begin{gathered}
36.000 \text { en nature de vase et de détritus } \\
\text { végétaux, }
\end{gathered}
$$

et 24.000 en nature de sables et graviers.

Le métré signale que le pourcentage de détritus végcitaux dans les vases est de $50 \%$ au moins (dont 3 à $5 \%$ de trones d'arbres et de branches de toutes dimensions). 
Le volume des dépôts ressort ainsi à $30 \mathrm{~m}^{3}$ par an et par km" de bassin rersant dont $9 \mathrm{~m}^{*}$ environ de débris végétaux.

C'est là un chiftre très faible comparé à ceux relevés sur les cours d'eau des Alpes :

Le Drae au Sautet $\left(1.000 \mathrm{~km} \mathrm{~km}^{2}\right)$ :

$350 \mathrm{~m}^{3}$ par an et par $\mathrm{km}^{2}$ de B.V.

Le Rhòne en amont du lac de Genève :

$650 \mathrm{~m}^{3} \mathrm{par}$ an et par $\mathrm{km}^{2}$ de B.V.

L'Arve à son confluent :

325 a 1.270 tonnes par an et par $\mathrm{km}^{2}$ de B.V. La Durance à Merindol (Vaucluse) :

750 tonnes par an et par $\mathrm{km}^{2}$ de B.V.
L'Isère à Grenoble $\left(5.720 \mathrm{~km}^{2}\right)$ :

530 tonnes par an et par km² de B.V.

Tous ces chiffres sont grossierement approximatifs; ils n'en confirment pas moins le très faible débil solide des cours d'eau du Massif Central par rapport à ceux des Alpes.

Signalons pour terminer que la tradition veut que, comme toutes les rivières descendant des Monts du Cantal, la Maronne roule des paillettes d'or; d'aucuns voient dans des noms de villages tels qu'Auriac un vestige de lindustrie des anciens orpailleurs de la haute Auvergne. Il semble bien établi que la ville d'Aurillac, sur les bords de la Jordanne, abrita autrefois de nombreux "batteurs d'or » qui tenaient peut-être leur malière première des rivières de la région.

\section{H P T TRE IX}

\section{QUELQUES DONNEES PHYSIQUES, CHIMIQUES ET BIOLOGIQUES}

\section{I. - Thermalisme et climatisme médicaux.}

Les eaux de la Maronne et de ses affluents coulant sur des terrains primitifs contiennent peu de matières dissoutes et présentent, en général, une réaction acide (P.H. $=7,2$ a 7,6 ) dont on a dû tenir compte pour la bonne conservation des bétons de barrage. Peu de sources thermales dans le bassin; signalons cependant celles de : Saint-Illide, aujourd'hui abandonnées, Saint-Martin-Valmeroux sur la Maronne, et la Bastide sur l'Aspres. Salers (altitude $1.000 \mathrm{~m}$ ) est déclarée station climatique, en raison de ses abords très dígagés qui favorisent l'ensoleillement et l'action vivifiante des vents venus des Monts du Cantal.

\section{II. - Pêche et ressources piscicoles du bassin.}

Le bassin de la Maronne est connu pour ses richesses piscicoles. Les eaux, riches en oxygène, ont un plancton abondant qui est, sans doute, à l'origine de leur couleur généralement brunâtre. La faune nutritive comporte surtout des larves d'eedyures et de phryganes très recherchées par les truites.

L'encaissement du lit et les nombreuses mouilles de la rivière et de ses affluents, constituent des endroits favorables aux frayères (le ruisseau de Carbonnières est réputé à ce point de vue). La truite est relativement abondante et attire de nombreux pêcheurs qui capturent aussi des chevennes, des ablettes, des goujons et exceptionnellement des perches du Canada. Quelques eyprins remontent de la Dordogne dans le cours inférieur de la Maronne où l'on rencontre également quelques moules d'ean douce.

Sur les affluents, tels que la Glane, le RiouTort, l'Encon, prospéraient, il y a quelques années, de belles colonies d'écrevisses, malheureusement en voie de disparition.

\section{CHA P T T R E X}

\section{UTILISATION DES EAUX}

\section{I. - Utilisations agricoles.}

Cours d'eau de montagne, drainnant une région très arrosée par les pluies d'origine atlantique, la Maronne n'a pas été mise à contribution pour irriguer des surfaces importantes.
On rencontre pourtant d'assez nombreuses prises suivies de petits canaux d'irrigation rustiques desservant les prairies qui couvrent les quelques épanouissements des vallées de la Maronne et de ses affluents (plaine de Salers, de Saint-Illide, de Rodomont, etc...). 


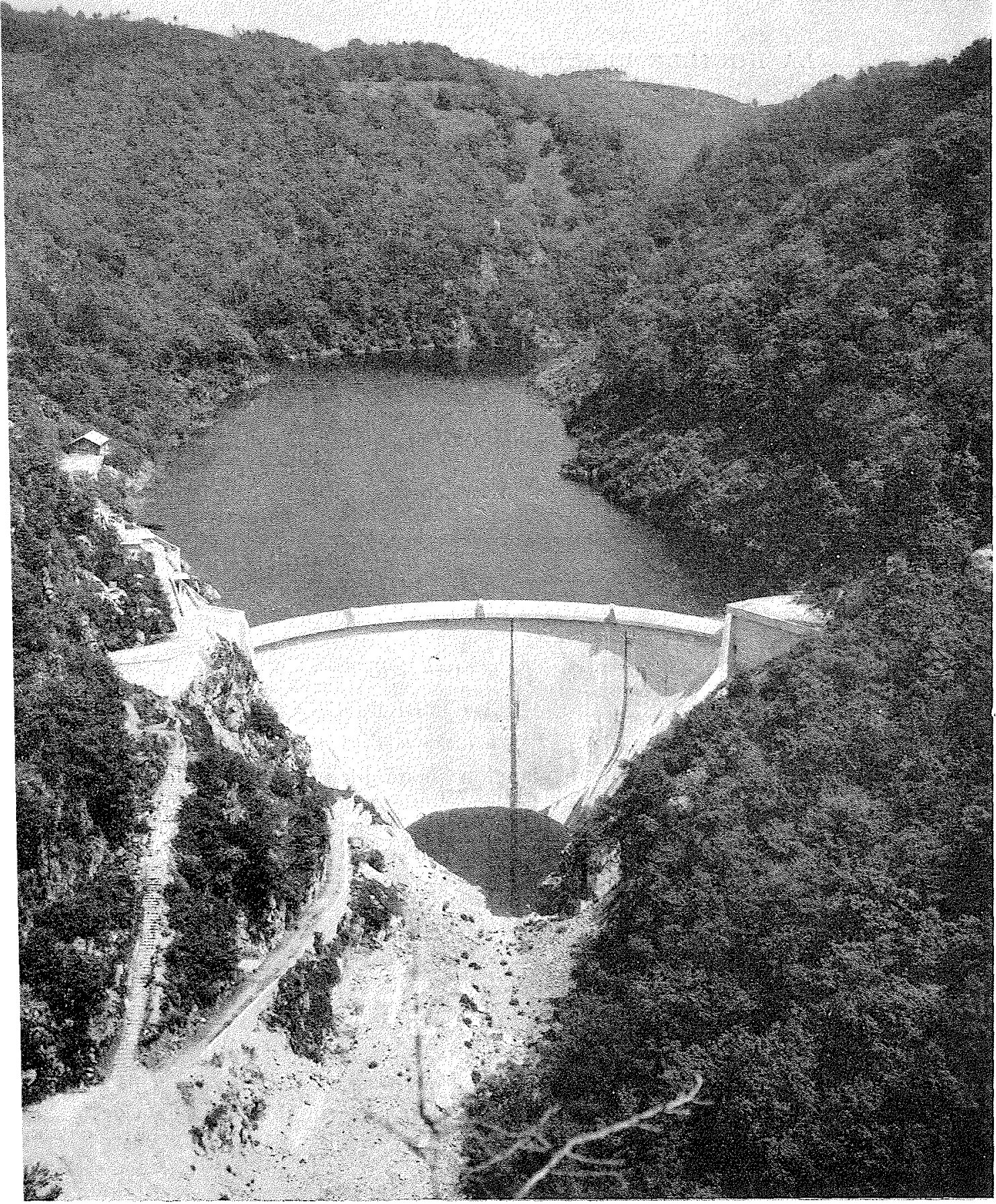

Photo $N^{\circ} 6$.

SaINT-Geniez-ò-Merte

(Corrèze)

LE BARRAGE DU Goun-NoIr.

Hauteur : $40 \mathrm{~m}$.

Longueur en crète : 99,5 mètres.

Capacité utile : $4, \overline{5} \times 10^{6} \mathrm{~m}^{3}$

\section{II. - Utilisations urbaines.}

Aucune ville ou agglomération urbaine importante ne se trouvant dans le bassin de la Maronne, aucune adduction d'eau potable ni aucun déversement d'eaux usées n'est à signaler.

\section{III. - Utilisations indutrielles.}

\section{a) Aménagements hydróllectrioues :}

Depuis des temps très reculés, les eaux de la Maronne et de ses affluents ont servi à actionner de petits moulins et des scieries utilisant, au moyen de roues à aubes, des chutes de 1 à
2 mètres environ (Moulins de Chaumiel, de Branzac, scierie de Saint-Rémy). En raison des difficultés d'accès et de l'importance des crues, il n'y a guère de moulins dans le cours moyen et inférieur de la Maronne; dans cette zone, les roues hydrauliques ont été établies sur des affluents (Glane, Riou-Tort, Encon), avant leur descente en cascatelles dans la gorge de la Maronne.

La première usine hydro-électrique importante fut celle de Saint-Paul qui produisait, vers le début du siècle, environ $500 \mathrm{~kW}$ en utilisant une chute de $55 \mathrm{~m}$ sur la haute Maronne; elle est aujourd'hui désaffectée.

Dès l'avènement de la Houille Blanche, la Ma- 


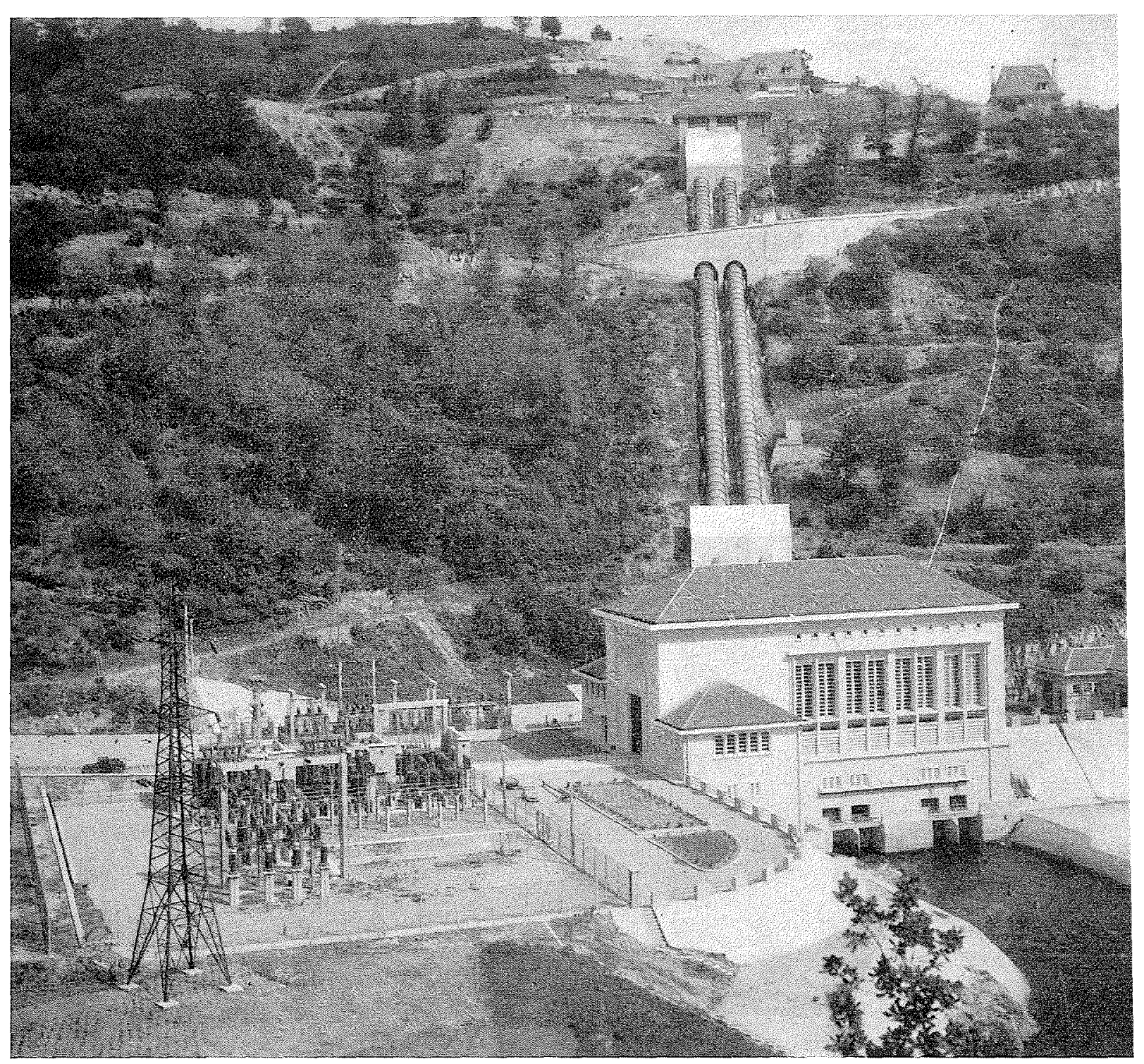

ronne, comme sa sour jumelle la Cère, attira l'attention des prospecteurs de chutes d'eau du Massif Central. Les grandes lignes de son aménagement se dégagèrent assez vite de l'examen de la morphologie de la rivière.

La forte alimentation spécifique de son haut Bassin fournit à son cours moyen des débits relativement importants s'écoulant dans les gorges à forte pente, creusées dans les dures granulites de Corrèze; le nombre des kilowatts sauvages par lilomètre de rivière est ici assez important pour que leur captage par des usines à longues dérivations souterraines, utilisant ingénieusement les caprices du tracé hydrographique, soit particulièrement avantageux. Par contre, dans la zone de micaschistes plus tendres, qui s'étend d'Espont au pont de l'Estouroc, la Maronne coule dans une vallée à faible pente qui comporte çà et là de larges épanouissements; la constitution d'un grand réservoir saisonnier dans cette région semble tout indiquée. L'aménagement du secteur de rivière à grand débit et de pente moyenne, compris entre les ruines de Merle et Argentat, pent $a$ priori être envisagé soit par usines-barráges, soit par dérivation.

De nombreux projets tentant d'utiliser au mieux ces heureuses dispositions naturelles fu- rent dressés dès 1918 par MM. Basiaux et DE Castelpers, puis par le Service des G.F.H. du Centre, par la Société des F.M. de la Vienne, par la Société Centrale pour l'Industrie Electrique, etc.. Ces études préliminaires aboutirent à la constitution en 1931 de la Société des F.M. de la Maronne qui commenca en 1940 les travaux de la chute de Saint-Geniez-ô-Merle et les poursuivit jusqu'en 1946, date à laquelle la nationalisation de l'Electricité les intégra au patrimoine d'Electricité de France.

Le plan d'aménagement des ressources hydroélectriques de la Maronne, actuellement en cours de réalisation, comprend les trois éléments suivants :

$1^{\circ}$ Le barrage-réservoir d'Enchanet, de $62 \mathrm{~m} \mathrm{de}$ hauteur de retenue, créant une réserve saisonnière de 73 millions de mètres cubes, suivi d'une « usine de pied » de $28.000 \mathrm{~kW}$ aménagée pour un débit de $55 \mathrm{~m}^{3} / \mathrm{s}$.

$2{ }^{\circ}$ La chute de Saint-Geniez, de $124 \mathrm{~m}$ de hauteur, comportant une réserve de compensation de 4,5 millions de mètres cubes, un canal de dérivation de $5.600 \mathrm{~m}$ et une usine de $34.000 \mathrm{~kW}$ aménagée pour un débit de $35 \mathrm{~m}^{3} / \mathrm{s}$. 
3" Le barrage de la Broquerie, de $51 \mathrm{~m}$ de hauteur de retenue, formant une réserve de compensation de 21 millions de mètres cubes, avec une usine de pied (1) de $22.000 \mathrm{~kW}$ aménagée pour un débit de $50 \mathrm{~m}^{3} / \mathrm{s}$.

Les figures 20 et 21 montrent que le plan d'aménagement utilise au mieux la configuration des lieux et les dispositions du profil en long de la riviere tels que nous les avons schematisés plus haut; les caractéristiques essentielles des trois usines qu'il comporte sont résumées dans le tableau ci-contre :

Les travaux d'aménagement de la chute de Saint-Geniez ont été entrepris en 1940; malgré les retards causés par les difficultés de tous ordres dues à la guerre, l'usine a pu ètre mise en service à la fin de l'année 1946, et débite sur le réseau francais depuis cette date.
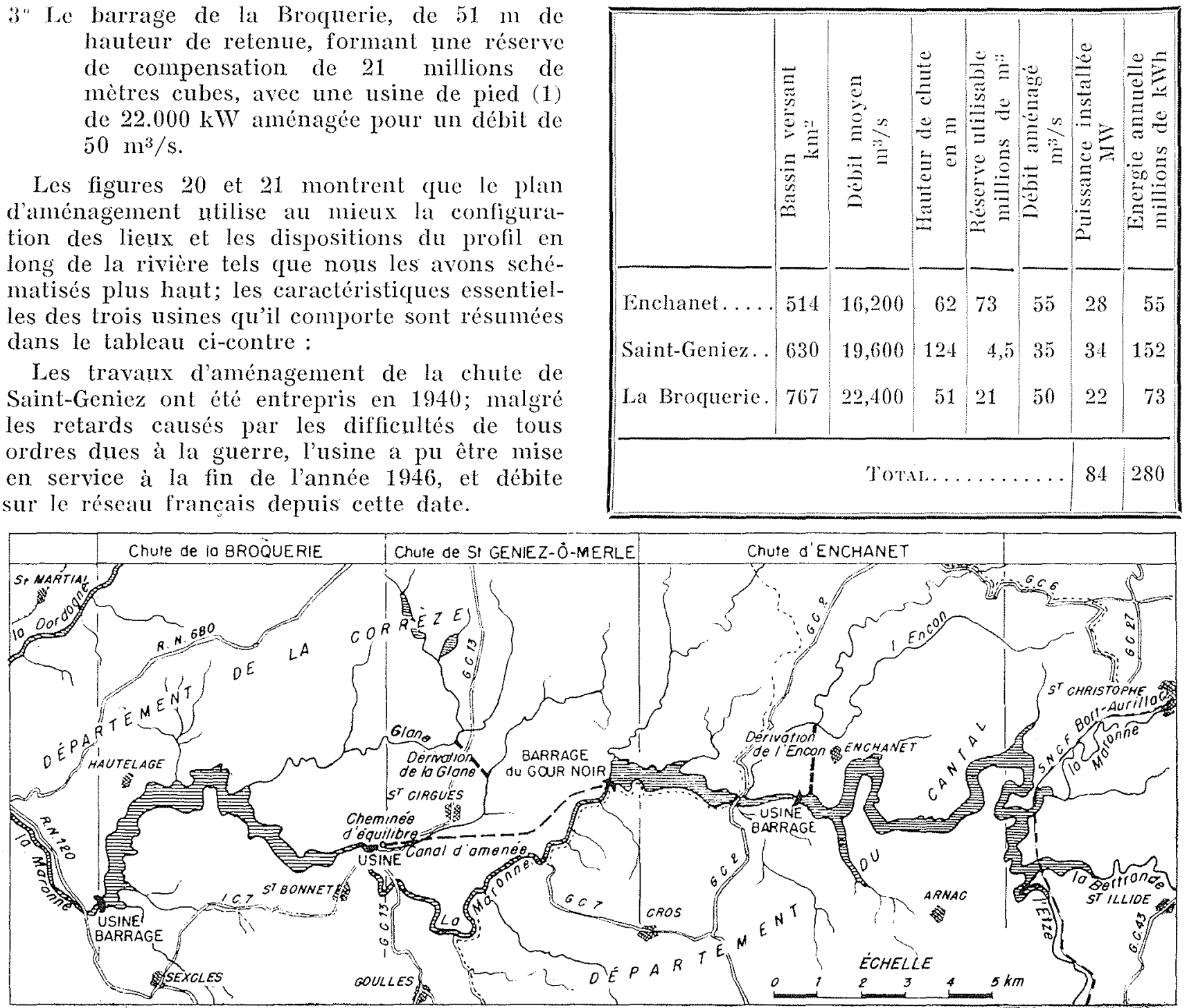

Fig. 20. - Bassin de la Maronne. - Aménagement hydroéle ctrique. Plan de situation des ouvrages.

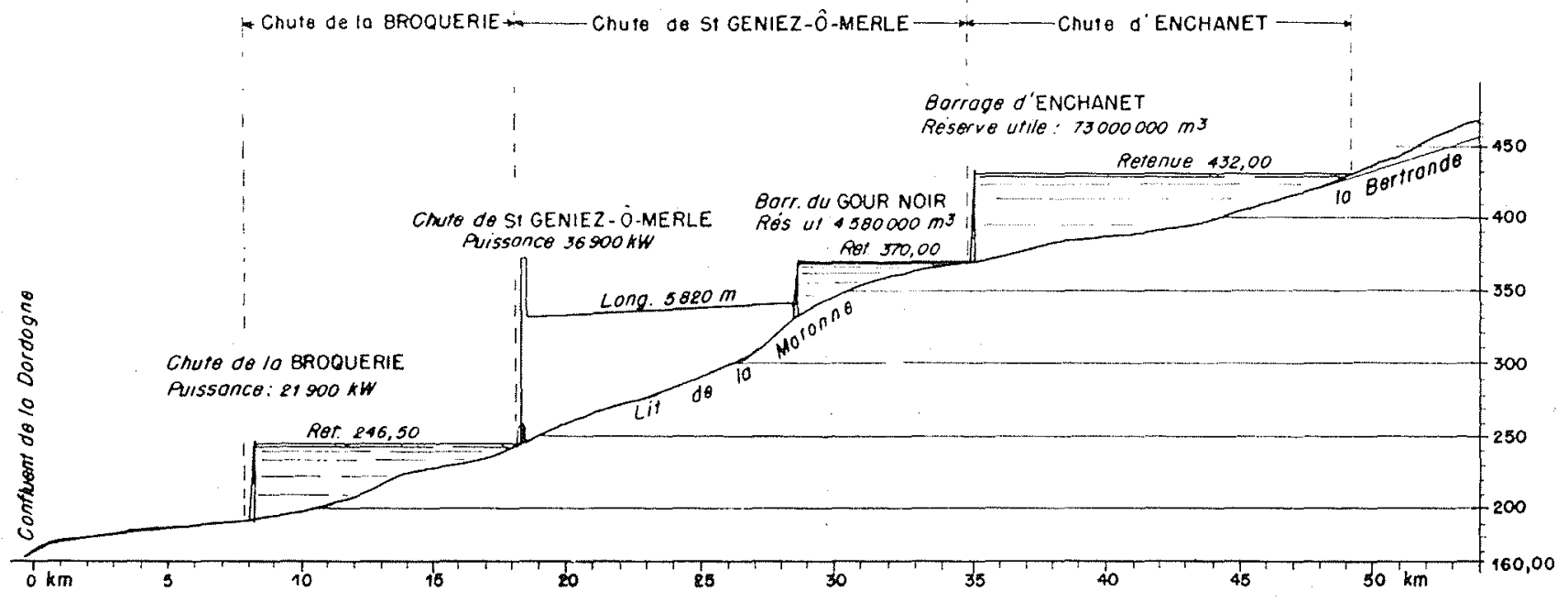

Fig. 21. - Bassin de la Maronne. - Aménagement hydroéle ctrique. Profil en long des ouvrages.

(1) En variante, on envisage une réserve alimentée par un canal d’amenée souterrain coupant la boucle de la Maranne au sud-ouest d'Hautelage. 


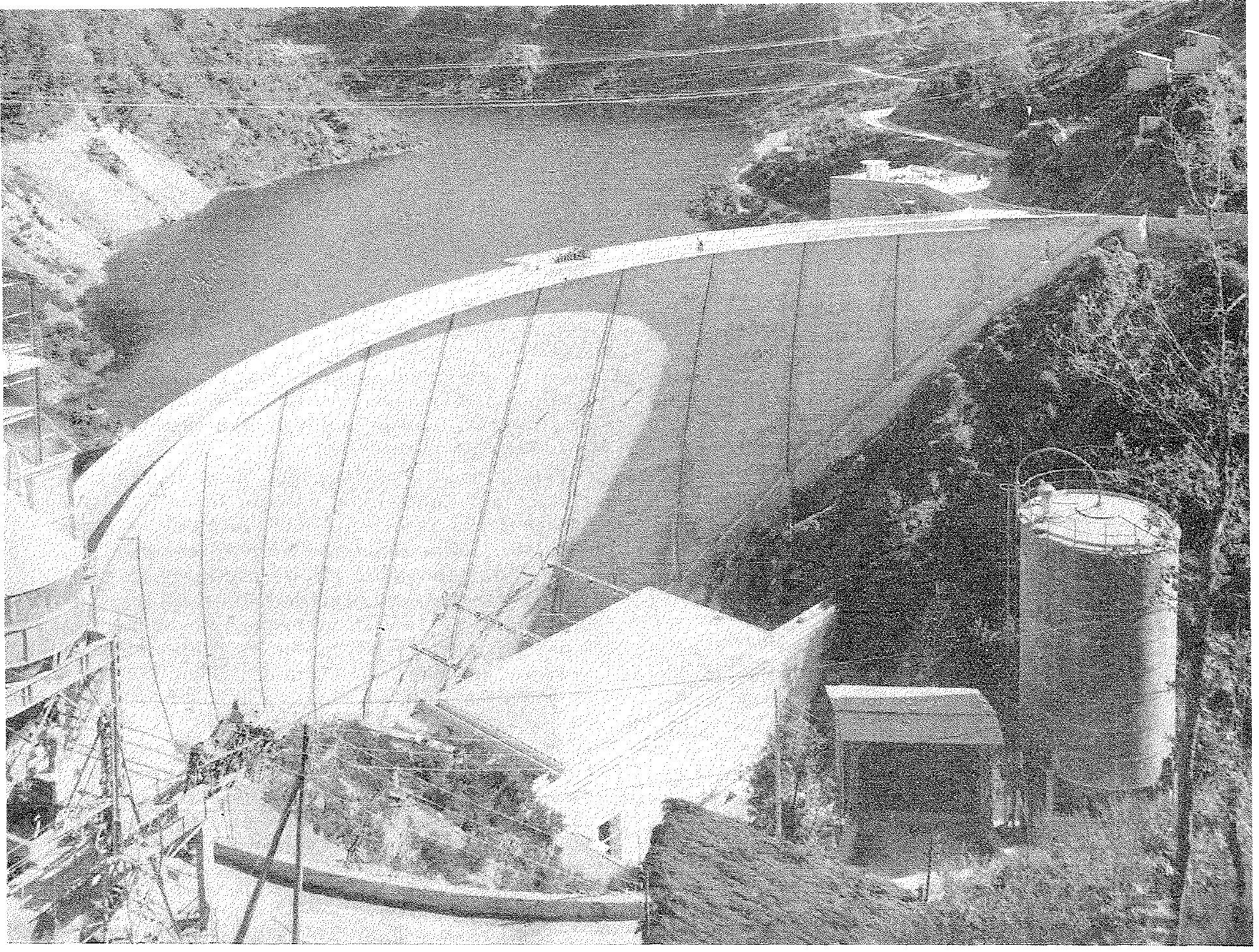

Photo No 8. - Le harrage D'ENchanet.

La main-d'œuvre et le matériel du chantier de Saint-Geniez ont alors été reportés sur les travaux du barrage et de l'usine d'Enchanet qui ont été mis en service au cours de l'année 1950.

La chute de la Broquerie devrait ètre exécutée a la suite pour parfaire l'aménagement de la Maronne ainsi régularisée par le réservoir saisonnier d'Enchanet.

La Société des F.M. de la Maronne avait étudié également un projet d'aménagement du haut bassin de la Maronne. Malgré leur pente souvent clevée, les rivières se prêtent ici moins bien à l'établissement d'usines hydro-électriques en raison de la dispersion des eaux entre la Maronme, la Bertrande et l'Etze. On peut envisager pourtant Ia création d'une chute d'environ $85 \mathrm{~m}$ en captant les eaux de la Maronne dans un barrage-réservoir à établir au droit de Sainte-Eulalie et en les déversant dans la Bertrande vers le conftuent du ruisseau de Besse où se trouverait une fenêtre d'attaque de la galerie de dérivation souterraine. Les eaux de la Bertrande et de la Maronne ainsi réunies pourraient être turbinées sous une chute de $80 \mathrm{~m}$ environ dans une deuxième usine utilisant la dénivellation comprise sur la Bertrande entre le confluent ci-dessus et la limite du remous du réservoir d'Enchanet. L'ensemble des deux usines aurait une capacité de production de 60 millions de $\mathrm{kWh}$ en année moyenne; elles exigeraient des investissements par kWh supérieurs de 50 à $100 \%$ à ceux correspondants à l'aménagement du cours moyen de la Maronne.

L'aménagement d'une chute de $140 \mathrm{~m}$ sur le Riou-Tort, entre la cuvette du Jaladis et la retenue du Gour Noir, donnerait 10 millions de kWh par an dans des conditions relativement avantageuses, mais la faible importance de cet écuipement en a fait différer la réalisation.

Des aménagements du mème ordre avaient été envisagés sur la Glane de Malesse et sur l'Encon, mais la dérivation maintenant réalisée de ces ruisseaux respectivement dans le canal d'amenée de Saint-Geniez, et dans la retenue d'Enchanet, leur enlève tout intérêt. Si la conception d'un ensemble de « microcentrales 》 à équipement normalisé et plus ou moins auto- 
matique, disséminées dans toute une région, devenait un jour une réalité, les prospecteurs trouveraient sur les petits affluents de la Maronne, de la Cère et de la haute Dordogne, de nombreux sites favorables; ceux ci se localisent dans la zone où les cours d'eau, quittant le plateau granitique sur lequel ils coulaient paresseusement, se précipitent en cascatelles dans les gorges profondes de ces grandes rivières.

\section{b) COMmunications ét tRansports fluviaux :}

Aucune section de la Maronne n'est classée parmi les rivières navigables ou flottabỉes; l'irrégularité des débits et les profils en lonğ accidentés ne permettent guère de l'utiliser aux transports par eau. Autrefois, le flottage des bois semblait pourtant avoir été pratiqué au voisinage d'Argentat.

\section{IV. - Utilisations touristiques.}

Les pittoresques gorges de la Maronne et de la Bertrande attirent chaque année quelques « canoëistes », La descente de ces eaux tumultueuses, encombrées de blocs et parsemées de rapides', n'est pas sans danger pour les débutants, ainsi qu'en témoignent deux accidents mortels survenus il y a quelques années.

Le haut bassin est parcouru durant la belle saison par de nombreux touristes venus admirer la cascade de Couderc sur la Maronne, la vallée de Fontanges sur l'Aspres, le curieux bourg médiéval de Salers, etc... Les gorges sauvages et peu accessibles des divers cours d'eau donnent aux adeptes de la pêche à la truite et aux écrevisses, l'impression - quelque peu fallacieuse de découvrir chaque jour de nouveaux terrains de chasse ou tout au moins de s'en assurer temporairement l'exclusivité.

Parmi les sites classés, une mention spéciale est due aux ruines d'un château féodal des $x r^{\circ}$ et XmI ${ }^{\circ}$ siceles, dites «les Tours de Merle». Campées sur un promontoire rocheux entouré par la Maronne (photo $n^{\circ} 5$ ) au voisinage de son confluent avec le ruisseau de Saint-Cirgues, eiles présentent un intérêt archéologique et touristique indéniable. Pour garder à cet ensemble sa sauvage grandeur, la cheminée d'équilibre de l'usine de Saint-Geniez-ô-Merle a été construite en souterrain et l'asséchement du lit de la Maronne, conséquence du fonctionnement de l'usine, a été évité par de petits seuils rusticues créant une série de miroirs d'eau autour du rocher austère qui porte les vestigès de la noble demeure d'Hughes de Merde et de ses filles.

\section{CONCLUSION}

Au terme de celte étude, c'est pour nous un agréable devoir de remercier tous ceux qui ont aidé à sa mise sur pied et à sa publication. Notre reconnaissance va d'abord à la Société des F.M. de la Maronne qui nous a autorisé, à la veille de la dernière guerre, à puiser dans ses dossiers les éléments qui nous étaient nécessaires et à l'Electricrté de France qui a facilité depuis, avec le même libéralisme, nos investigations. Mais c'est à la Société Hydrotechnioue de France que notre travail doit d'avoir vu le jour : le « Prix de Monographie Hydrologique » pour l'année 1949 qu'elle a bien voulu lui attribuer a été créé sur l'initiative de son regretté Président, M. Milon, appuyé par M. Barrillon, Membre de l'Institut et Président de son Comité Technique, et par M. Emmanuel DE MARTonne, Membre de l'Institut et Président de la Commission d'Hydrologie Statistique. Nous remercions ces hautes personnalités pour les adjonctions et corrections - dont nous avons tenu le plus grand compte - qu'elles nous ont suggéré d'apporter à notre texte initial.

Le rassemblement des données statistiques qui forment la base de notre étude n'a pu être mené à bien que grâce à l'obligeance de nombreux ingénieurs des Services publics; qu'ils veuillent bien trouver ici nos vifs remerciements; ceux-ci s'adressent en particulier à M. BARrì̀re,

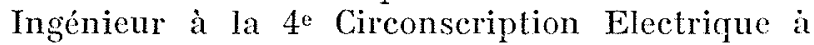
Limoges, et à M. Ivanoff, Chef du Contrôle du Chantier d'Enchanet à Electricité de France, qui nous ont fourni une précieuse documentation sur les débits et les températures dans le bassin de la Maronne.

Sous sa forme actuelle, notre étude résulte de la mise à jour d'un essai de monographie hydrologique rédigé en 1932 par l'un de nous, alors Ingénieur à la Société des F.M. de la Maronne, afin de rassembler et de coordonner les diverses données expérimentales recueillies en vue d'étudier l'aménagement hydro-électrique de cette rivière. Nous serions heureux si notre tentative incitait les nombreux ingénieurs, engagés aujourd'hui dans l'exploitation de la Houille Blanche, à dresser la monographie hydrologique des cours d'eau qui leur sont familiers. 
A l'occasion des nombreuses courses sur le terrain qu'exige leur passionnant métier, ils auront décelé, par observation personnelle ou par ouï-dire, bien des particularités non seulement du régime des températures, des pluies et des débits, mais aussi de la nature du sol, et de sa couverture végétale; cette expérience intime du bassin les préparera à méditer les travaux de leurs maîtres et de leurs aînés, et à les appliquer à l'interprétation toujours si délicate des données disponibles. Ils se doivent d'apporter leur contribution à une connaissance plus exacte de nos belles rivières de France.

Chemin faisant, sans doute seront-ils conquis par l'agreste poésie des eaux et, avec l'un des maîtres incontesté de l'Hydrologie francaise, M. Aimé Coutagne, - auquel notre travail doit tant, - ils diront : ... puiqu'il faut,

Pour créer du profit, que de la Beauté meure, J'irai vous contempler', vous écouter plus haut, Flots vierges, non captés, dont la chanson demeure.

\section{B I B L I O G R A P H I E}

Coutage (A). - Monographie hydrologique du bassin du Bés.

(Paris, Compte rendu des travaux de la Sociélé Hydrotechnique de France, $\mathrm{n}^{\circ} 69,15$ octobre 1932.$)$

- Hydrométéorologie des bassins de haute montagne.

(Paris, Société Hydrotechnique de France, octobre 1942.)

Fischer. - L'Adour et ses affluents. (Paris, Hachette, 1929.)

Gimrat (R.). -- Aménagement hydro-électrique des cours d'eau. - Statistique et calcul des probabilités.

(Paris, Revue Générale de l'Electricité, 22 octobre 1932.)

- Aménagement hydroélectrique des cours d'eau. Statistique mathématique et probabilités.

(Paris, Revae Générale de l'Hydralique, $\mathrm{n}^{\circ} 60$, marsavril 1937 , pages $853 \mathrm{H}$ ì $861 \mathrm{H}$.)

LuGEON. - Précipitations atmosphériques, éconlements et hydro-électricité. (Paris, Dunod, 1928.)
De Martonne (E.). - Géographie physique : principalement chapitres V et VI. Massif Central. (Paris, Librairie Armand Colin, 1942.)

Mazlocm (S.). - L'Afrique. Etude hydrologique.

(Paris, Revue de Géographie physique, 1939.)

Mroos (Mlle Françoise). - Le Lae d'Annecy. - Etude géographique.

(Paris, Annales de Géographie, 1939, pages 120 à 137 , 11 figures.)

Parite (M.). - Cours de Potamologie.

(Grenoble, Ecole des Ingénieurs Hydrauliciens de Grenoble, 1949.)

Renaud (A.). - La chute de Saint-Geniez et le barrage du Cour-Noir, sur la Maronne.

(Paris, la Technique des Travaux, $\mathrm{n}^{\circ}$ 11-12, novembredécembre 1948 , pages 339 à 377 .)

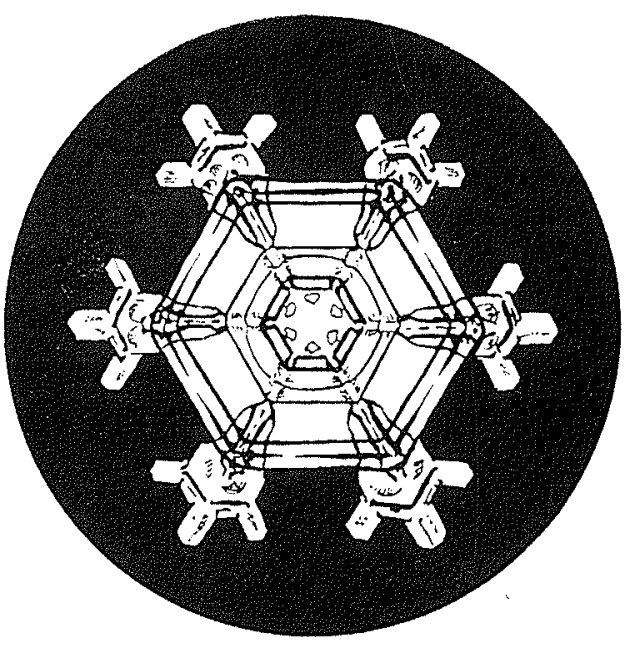

